\title{
A Pilot Study of the Effects of an Australian Centre-Based Early Intervention Program for Children with Autism
}

\author{
Jessica Paynter ${ }^{*}$ James Scott, Wendi Beamish, Michael Duhig and Helen Heussler
}

AEIOU Foundation, N77 Recreation Road, NATHAN, QLD 4111, Australia

\begin{abstract}
The current study sought to evaluate the effectiveness of an Australian centre-based early intervention program for children with autism. Outcomes for 10 children with autistic disorder aged between 32 and 65 months of age participating in the AEIOU early intervention program were investigated. Measures of educational, cognitive, and adaptive skills as well as autism symptoms were administered. Significant gains in educational skills in the areas of cognitive verbal/preverbal, fine motor and visual-motor imitation, motor domain score, and social reciprocity, were obtained as were decreases in autism symptoms. Limited evidence of gains was obtained for measures of cognitive or adaptive behaviour skills. This study provides promising preliminary evidence in support of the AEIOU program in terms of symptom reduction and increases in educational skills. Limitations and future research directions are discussed.
\end{abstract}

Keywords: Autism spectrum disorder, early intervention, educational skills, outcome.

\section{INTRODUCTION}

Autism Spectrum Disorders (ASD) including Autistic Disorder, Asperger Disorder, and Pervasive Developmental Disorder - Not Otherwise Specified [1] are characterised by qualitative impairments in communication, social relations, and repetitive and restricted behaviours and interests [2]. These disorders affect approximately one percent of children [3-6]. ASD can be reliably identified and diagnosed in very young children between the ages of 2 to 4 years [7-9]. The characteristic triad of impairments, together with difficulties in emotional processing, sensory-perceptual processing, and motor proficiency [10], adversely affect developmental trajectories and family functioning in the early years. Impact routinely continues across the lifespan influencing independence, academic success, and participation in school, work, and community life [11]. In addition, adolescents and adults with ASD are at high risk of psychiatric comorbidity with an associated need for ongoing support $[12,13]$.

Early intervention has been proposed as an effective means for reducing future disability in children with ASD [14]. Australian Best Practice guidelines for early intervention [14] suggest that intervention should commence as early as possible following diagnosis (ideally between $2-4$ years), use multidisciplinary assessment, be individualised and multidisciplinary, and have an autism-specific focus for at least 20 hours per week. Interventions should be delivered across settings (e.g. centre-based, home-based) with a high degree of structure and low staff to child ratio (maximum of 2-4 children per adult), and employ a functional approach to challenging behaviours. These interventions should emphasise family collaboration and include transition support out of the program [14].

\footnotetext{
*Address correspondence to these authors at the AEIOU Foundation, N77 Recreation Road, NATHAN, QLD 4111, Australia;

Tel: +617 3320 7924; Fax: +617 3277 2422;

E-mails: jessica.paynter@aeiou.org.au, Jessica.paynter@me.com
}

At this point in time, intervention services vary substantially in theoretical orientation, intervention focus and intensity, context for delivery, degree of family involvement, and efficacy evidence [15]. There is a need for ongoing research into early intervention programs [16]. Although there has been substantial research into Early Intensive Behavioural Interventions (EIBI), research into other models of intervention has been rare. For example, despite "best practice" guidelines, as described above, recommending the use of educationally-based interventions, the efficacy of these types of interventions has typically only been investigated in the context of control conditions in EIBI trials where they have received little attention in their own right. Clearly there is a need to specifically evaluate their merit. As such, educationally-based autism-specific early intervention programs will be reviewed as context to the present study.

Educationally-based autism-specific early intervention programs have often been described as "eclectic" [17] or "generic" [18]. This is because these programs typically do not subscribe to a single program, philosophy, or theoretical approach, but instead aim to be comprehensive and offer a range of teaching strategies such as Picture Exchange Communication Systems [19], activities drawn from the Treatment and Education of Autistic and Related Communication Handicapped Children (TEACCH) [20], and positive behaviour support [21]. Examples of programs which have received attention are special nursery placement $[22,23]$, autism-specific nursery [24], eclectic autismspecific preschools [25], eclectic-developmental autismspecific preschools [26], and autism-specific primary schools or units for children under six years [27]. These programs share in common an autism-specific focus, structuring their teaching in nursery, preschool, or kindergarten classrooms, and incorporate elements of educational programs such as circle time, individual education plans, and a positive approach to challenging behaviour. These programs tend to be delivered by multidisciplinary teams in which teachers 
coordinate classroom activities and intervention is actively supported by speech pathologists, psychologists, and/or occupational therapists.

Key outcomes investigated across studies have included educational and cognitive skills, as well as adaptive behaviour, and autism symptomotology (see Table 1). This body of research has found some evidence of gains over time for children in terms of educational and cognitive skills, adaptive behaviour, and autism symptoms. These data suggest that intervention of this kind may lead to improvements in these areas. However, there is clearly a need for further research into specific programs.

The present research focuses on the AEIOU program. AEIOU is a not-for-profit organisation in Queensland, Australia that provides intensive early intervention to children from age $2 \frac{1}{2}$ to 6 years who have been diagnosed with an Autism Spectrum Disorder (ASD). To date, there has not been an empirical investigation into the effectiveness of the AEIOU program although data have been collected on individual children as an integral part of their intervention.

The AEIOU program is based on Australian Best Practice Guidelines for early intervention for children with autism spectrum disorders developed by Roberts and Prior [28]. A blend of evidence-based strategies drawn from established treatments and interventions are used. Strategies include the antecedent package, behavioural package, behavioural treatment, joint attention intervention, modelling, naturalistic teaching strategies, pivotal response treatment, schedules, self-management, and story-based interventions [e.g., 29]. Two augmentative communication systems are used; the Picture Exchange Communication System [19] and a modified sign language using key signs drawn from Australian Sign Language. The combination of strategies used with each child is based on his/her unique needs and strengths as determined by comprehensive multidisciplinary assessment.

Staff include one psychologist, one research fellow, speech therapists, occupational therapists, early childhood teachers, and childcare professionals. The classrooms have a 1:2 to 1:4 staff to child ratio. The full-time program involves a minimum of 25 hours per week of direct and intensive intervention. The centre also functions as a long day-care outside of these hours and is open from $7.00 \mathrm{am}$ to $5.00 \mathrm{pm}$ five days per week for 48 weeks per year. Intensive programming occurs for 40 weeks per year.

The AEIOU curriculum is designed to support the development of each child in four key areas of learning and development: Social Emotional; Language and Communication; Physical; and Cognitive. An Individual Education Plan (IEP) is used to individualise intervention to each child. Teaching occurs within the contexts of classroom routines and activities (e.g., free play, learning centres, circle/mat times, snack, and toileting). Speech, occupational, and psychological therapy consultation and support are provided in each classroom.

The program includes a family education component that consists of regular parent training by allied health and teaching staff. Training covers a range of topics including

Table 1. Outcomes of Previous Studies of Autism-Specific Early Learning Programs

\begin{tabular}{|c|c|c|c|}
\hline Domain & Measure* & Studies & Results (Pre/Post within Groups Comparisons) \\
\hline $\begin{array}{l}\text { Educational } \\
\text { Skills }\end{array}$ & PEP-R & $\begin{array}{l}\text { Reed et al. [22] } \\
\text { Reed et al. [23] }\end{array}$ & $\begin{array}{l}\text { - Significant improvement for "special nursery placement" on gross motor, cognitive } \\
\text { and verbal subscales } \\
\text { - Significant improvement for "Autism-specific special nursery" on the overall PEP-R } \\
\text { score }\end{array}$ \\
\hline \multirow[t]{2}{*}{$\begin{array}{l}\text { Cognitive } \\
\text { Skills }\end{array}$} & BAS- II & Reed et al. [22] & $\begin{array}{l}\text { Significant improvement for "special nursery placement" on picture matching, naming, } \\
\text { and early number skills subscales }\end{array}$ \\
\hline & MSEL & $\begin{array}{l}\text { Zachor \& Ben } \\
\text { Itzchak. [25] }\end{array}$ & $\begin{array}{l}\text { - Significant raw scores gains across all four domains for an "eclectic-developmental" } \\
\text { autism-specific preschool program on } \\
\text { - } \\
\text { Gains were significant in standard scores on receptive language only }\end{array}$ \\
\hline \multirow[t]{4}{*}{$\begin{array}{l}\text { Adaptive } \\
\text { Behaviour }\end{array}$} & VABS-Screener & Charman et al. [27] & $\begin{array}{l}\text { Significant changes over time on the VABS Screener on domain age-equivalent scores } \\
\text { but no significant difference in the overall adaptive behaviour composite score. }\end{array}$ \\
\hline & \multirow[t]{3}{*}{ VABS } & Reed et al. [23] & $\begin{array}{l}\text { Children attending an "Autism-specific special nursery" school significantly improved } \\
\text { on composite score }\end{array}$ \\
\hline & & Magiati et al. [24] & $\begin{array}{l}\text { Significant increases in mean age-equivalent scores on the VABS for "Autism-specific } \\
\text { special nursery" group }\end{array}$ \\
\hline & & $\begin{array}{l}\text { Zachor \& Ben } \\
\text { Itzchak [25] }\end{array}$ & $\begin{array}{l}\text { - } \\
\text { - } \quad \text { Significant gains in each of the four raw domain scores of adaptive behaviour } \\
\text { scores } \\
\text { - } \quad \text { Significant decrease of motor skills standard scores }\end{array}$ \\
\hline \multirow[t]{2}{*}{$\begin{array}{l}\text { Autism } \\
\text { Symptoms }\end{array}$} & ADOS & Zachor et al. [26] & $\begin{array}{l}\text { Significant gains on the social interaction domain score for the "eclectic- } \\
\text { developmental" intervention group }\end{array}$ \\
\hline & SCQ & Charman et al. [27] & $\begin{array}{l}\text { No significant changes over time for the measure of autism symptoms on the Social } \\
\text { Communication Questionnaire }\end{array}$ \\
\hline
\end{tabular}

* PEP-R: Psychoeducational Profile- Revised; BAS-II: British Abilities Scale- II; MSEL: Mullen Scales of Early Learning; VABS: Vineland Adaptive Behaviour Scale; ADOS Autism Diagnostic Observation Scale; SCQ: Social Communication Questionnaire. 
play skills, managing challenging behaviour, transitions, and communication strategies. Parents are also able to access home-visits by members of the team to assist with generalisation of strategies to the home. Parent involvement is a critical part of the program, particularly in relation to goal setting and review, the development of positive behaviour supports, and the transition process.

The present study is based on standardised assessments (described below) and aims to investigate the effectiveness of this intensive early intervention program through evaluating changes in children's intellectual, educational, and adaptive functioning, together with level of autistic symptoms. Based on previous research into educationallybased interventions, we hypothesised that improvements would be seen in children's intellectual, educational, and adaptive functioning as well as their level of autistic symptoms following a year of intervention. These hypotheses were examined through evaluating changes in assessment scores collected for the first cohort of children exiting the program by July 2011 .

\section{MATERIALS AND METHODS}

\section{Institutional Consent}

Ethics approval was granted by Griffith University and gatekeeper approval was given by the AEIOU Foundation. Signed informed consent was obtained from parents of participating children.

\section{Participants}

This study is focused on children who entered the AEOIU program in February 2010 and completed their placement by July 2011. Eligibility for program entry is based on a DSM-IV diagnosis [30] of an autism spectrum disorder (ASD) by a medical practitioner (paediatrician, child psychiatrist, or neurologist) combined with a chronological age at intake between 30 and 71 months. In addition, all children included in this study had an SCQ [31] score greater than 11 as recommended by Lee et al., [32], $M=17.10, S D=3.21$, Range 13-23. From a possible 13 children, 10 of their parents (77\%) signed consent for the data collected while in the program to be used for this evaluation. All ten children had been diagnosed as having autistic disorder. The participants ( 9 males, 1 female) attended the AEIOU program for a mean duration of 11.89 months, $S D=2.83$ (8.77-16.36). Mean age at program entry was 53.93 months ( $S D=11.43$, range 32.43 to 65.97 months) and mean age at program exit was 68.22 months $(S D=9.12)$. Over the course of the program, the majority of children (9/10) attended on a full-time basis (minimum 25 hours per week) with one child attending on a part-time basis (five day fortnight, average of 13 hours per week). During each child's final term (approximately 12 weeks in duration), attendance was gradually reduced because children spent increasing time at their next educational setting. The majority of parents were married $(6 / 10)$, with a subset divorced $(2 / 10)$, single $(1 / 10)$, or widowed $(1 / 10)$. The majority of parents were English speaking Caucasian (7/10); however a subset came from a culturally and linguistically diverse background (3/10).

\section{Measures and Procedure}

Standardised assessments of educational (PEP-3) and cognitive skills (MSEL) were conducted by the lead author who has extensive experience in assessing children with ASD and is not involved in the daily AEIOU program. Time 1 child assessments were completed within 4 months of each child commencing the program. The PEP-3 was completed within the first 4 weeks $(M=13.30$ days, $S D=9.11)$, and the MSEL was added later (at 3 months; MSEL, $M=102.30$ days, $S D=17.46$ ). The late addition of the MSEL was due to changes in assessment requirements linked to the centre's funding. Time 2 follow-up assessments were conducted after 12 months or on exit (whichever came first) for both measures, which resulted in a shorter follow-up period for the MSEL $(M=222.00$ days, $S D=51.75)$ than the PEP-3 $(M=297.30$ days, $S D=59.25)$.

Educational skills. The PEP-3 [33] is a standardised assessment of communication, motor skills and behaviours specifically designed for children with ASD who have a development age between 6 months and 7 years. The 10 performance subtests were administered to children in the present study.

Cognitive skills. The Mullen Scales of Early Learning [34] is an individually administered assessment of language, motor and perceptual abilities of children from birth to 68 months. It can be used with older children as in the present study where mental age is lower than chronological age. This measure was used to assess a range of skills in children with autism in previous research [25, 35-37]. Age-equivalent scores were used in the analyses as some children's chronological age exceeded 68 months at post (Time 2) assessment; additionally many children did not reach the basal for standard scores.

Questionnaire assessment was used to assess the level of ASD symptoms children displayed as well as to measure their adaptive behaviours. The Social Communication Questionnaire (SCQ [31]; previously titled the Autism Screening Questionnaire [38]) was completed at intake and at exit or 12-months, while the Vineland Adaptive Behaviour Scales-II (VABS-II [39]) was completed at Time 1 after approximately 3 months by parents and teachers (VABS-P, $M=108.00$ days, $S D=11.23$; VABS-T, $M=103.80$ days, $S D=16.46$, respectively) due to changes in assessment requirements linked to the centre's funding.

Autism symptoms. The SCQ is a short 40-item questionnaire derived from the Autism Diagnostic Interview-Revised [40]. On this questionnaire, parents indicate whether a child displays characteristic autistic behaviours. Verbal/language items can be omitted for children who are non-verbal. A total score from $0-32$ or 39 (depending on a child's language level) is calculated; higher scores indicate greater severity of symptoms and greater likelihood of an autism diagnosis. Clinical cut-offs have been developed to identify children with probable autism (cut-off $=22$ ) or all ASD (cut-off $=15$ ) [38]. More recent research suggests a lower cut-off of 11 [32].

Adaptive skills. The VABS-II [39] parent-caregiver form and teacher report forms were completed by parents and teachers of children at AEIOU, respectively. Both of these measures assess adaptive behaviour in four domains: communication, daily living skills, socialisation, and motor skills. Items are rated as $2=$ usually, $1=$ sometimes or partially, $0=$ never. Raw scores were converted into age- 
equivalents and standard scores using tables in the manual. Age-equivalent scores were used as the main unit of analysis in the present study as recommended by previous research for measuring the developmental level of young or lowfunctioning children with ASD [41]. The VABS-II is acknowledged as a valid measure for the assessment of children with ASD and is the most widely used assessment tool to measure adaptive skills in this population [42].

\section{RESULTS}

\section{Child Outcomes}

Educational skills. Table 2 presents standard scores on the PEP-3. Standard scores increased from Time 1 to Time 2 on each of the domains and on most subdomains (with characteristic verbal behaviours similar over time) indicating improvements in skills over time. Significant improvements over time were detected on both the maladaptive behaviour and motor skills domains with medium to large effects respectively. Improvements on the communication domain approached significance $(p=0.07)$ with a medium effect. Significant improvements over time were detected in cognitive verbal/preverbal, fine motor, visual-motor imitation, and social reciprocity subdomains with a large $(r>0.60)$ effect.

Cognitive ability. Table 3 presents age-equivalent domain scores on the MSEL. A pattern of improvements over time was observed and there was a medium to large effect. Age-equivalents were used as the main unit of analysis as the majority of children $(>50 \%)$ did not reach the basal t-score on each scale. The difference between Time 1 and 2 scores was significant for the receptive language scale only. However, it should be noted that ceiling effects were obtained on this measure, particularly on the visual reception scale where some children achieved a near-perfect score.

Autism symptoms. The average SCQ total score moved from the clinical range $(M=16.63, S D=2.72)$ to the subclinical range at follow-up $(M=9.63, S D=4.24)$, which was a significant reduction in ASD symptoms with time, $t$ $(7)=4.17, p=.004, r=.84$.

Adaptive behaviour: Parent ratings. Table 4 presents standard scores showing improvement over time with a medium effect for communication, daily living skills, and socialisation domains. Yet, these changes were not significant. Standard scores for motor skills decreased, although this change over time was not significant. Significant gains in age-equivalent scores were observed in expressive and written communication subdomains as well as fine motor skills with large effects.

Teacher ratings. Consistent with parent ratings, standard scores on teacher ratings improved over time in communication, daily living, and socialisation domains although none of these changes in scores over time reached traditional significance (see Table 5). Communication showed a medium effect. Motor skills also improved with time and showed a medium effect. In terms of age-equivalent subdomain scores, significant improvements were observed in written and academic areas with a large effect.

Table 2. Psychoeducational Profile Standard Scores (ASD Norms)

\begin{tabular}{|c|c|c|c|c|c|c|}
\hline & Time 1 (SD) & Time 2 (SD) & $t$ & $d f$ & $p$ & $r$ \\
\hline Communication Domain $^{\prime}$ & $\begin{array}{l}32.80 \\
(9.10)\end{array}$ & $\begin{array}{l}36.10 \\
(9.67)\end{array}$ & 2.03 & 9 & .07 & .56 \\
\hline Cognitive Verbal/Preverbal & $\begin{array}{l}11.10 \\
(3.50)\end{array}$ & $\begin{array}{l}12.30 \\
(3.02)\end{array}$ & 4.13 & 9 & $<.01 * *$ & .81 \\
\hline Expressive Language & $\begin{array}{l}11.00 \\
(3.50)\end{array}$ & $\begin{array}{l}12.20 \\
(3.91)\end{array}$ & 1.56 & 9 & .15 & .46 \\
\hline Receptive Language & $\begin{array}{l}10.70 \\
(3.27)\end{array}$ & $\begin{array}{l}11.60 \\
(3.03)\end{array}$ & 1.22 & 9 & .25 & .38 \\
\hline Motor Skills Domain & $\begin{array}{l}29.30 \\
(6.43)\end{array}$ & $\begin{array}{l}33.60 \\
(6.36)\end{array}$ & 5.55 & 9 & $<.01 * *$ & .88 \\
\hline Fine Motor & $\begin{array}{c}9.50 \\
(2.37)\end{array}$ & $\begin{array}{l}11.40 \\
(1.96)\end{array}$ & 3.94 & 9 & $<.01 * *$ & .80 \\
\hline Maladaptive Behaviours ${ }^{+}$ & $\begin{array}{c}42.70 \\
(11.54)\end{array}$ & $\begin{array}{l}46.70 \\
(9.68)\end{array}$ & 1.36 & 9 & .21 & .41 \\
\hline Social Reciprocity & $\begin{array}{l}10.10 \\
(2.60)\end{array}$ & $\begin{array}{l}11.80 \\
(2.04)\end{array}$ & 2.68 & 9 & $.03 *$ & .67 \\
\hline Characteristic Motor Behaviours & $\begin{array}{l}10.50 \\
(3.34)\end{array}$ & $\begin{array}{l}11.60 \\
(2.27)\end{array}$ & 1.18 & 9 & .27 & .36 \\
\hline Characteristic Verbal Behaviours & $\begin{array}{c}9.60 \\
(4.55)\end{array}$ & $\begin{array}{c}9.50 \\
(5.06)\end{array}$ & -0.07 & 9 & .95 & .02 \\
\hline
\end{tabular}


Table 3. Mullen Scales of Early Learning Age-Equivalents

\begin{tabular}{|l|c|c|c|c|c|c|}
\hline \multicolumn{1}{|c|}{ Scale } & $\begin{array}{c}\text { Time 1 } \\
\text { (SD) }\end{array}$ & $\begin{array}{c}\text { Time 2 } \\
\text { (SD) }\end{array}$ & $\boldsymbol{t}$ & $\boldsymbol{d}$ & $\boldsymbol{r}$ \\
\hline \hline Visual Reception & $\begin{array}{c}43.00 \\
(16.94)\end{array}$ & $\begin{array}{c}51.11 \\
(23.83)\end{array}$ & 1.49 & 8 & .17 \\
\hline Fine Motor & $\begin{array}{c}38.33 \\
(14.41)\end{array}$ & $\begin{array}{c}41.33 \\
(14.21)\end{array}$ & 1.27 & 8 & .24 & .47 \\
\hline Receptive Language & $\begin{array}{c}31.78 \\
(15.06)\end{array}$ & $\begin{array}{c}37.00 \\
(16.32)\end{array}$ & 2.93 & 8 & $.02 *$ & .72 \\
\hline Expressive Language & $\begin{array}{c}29.33 \\
(12.61)\end{array}$ & $\begin{array}{c}36.11 \\
(13.04)\end{array}$ & 1.85 & 8 & .10 & .55 \\
\hline
\end{tabular}

$* p<.05$.

Interpersonal relationship skills also trended $(p=.06)$ towards significance, with a medium to large effect. All other areas showed non-significant changes over time.

\section{DISCUSSION}

Outcome data from this pilot study adds to the growing body of research into early intervention for children with autism conducted in early childhood education and care environments It also provides preliminary support for the AEOIU program. Promising results were obtained in the educational measure and measures of autism symptoms and point to positive outcomes for children who attend this service. Limited evidence of gains (age-equivalent gains only) was obtained for the cognitive and adaptive behaviour measures.

Table 4. VABS-II: Parent/Caregiver Rating Scale: Age-Equivalents (AE) and Standard Scores (SS)

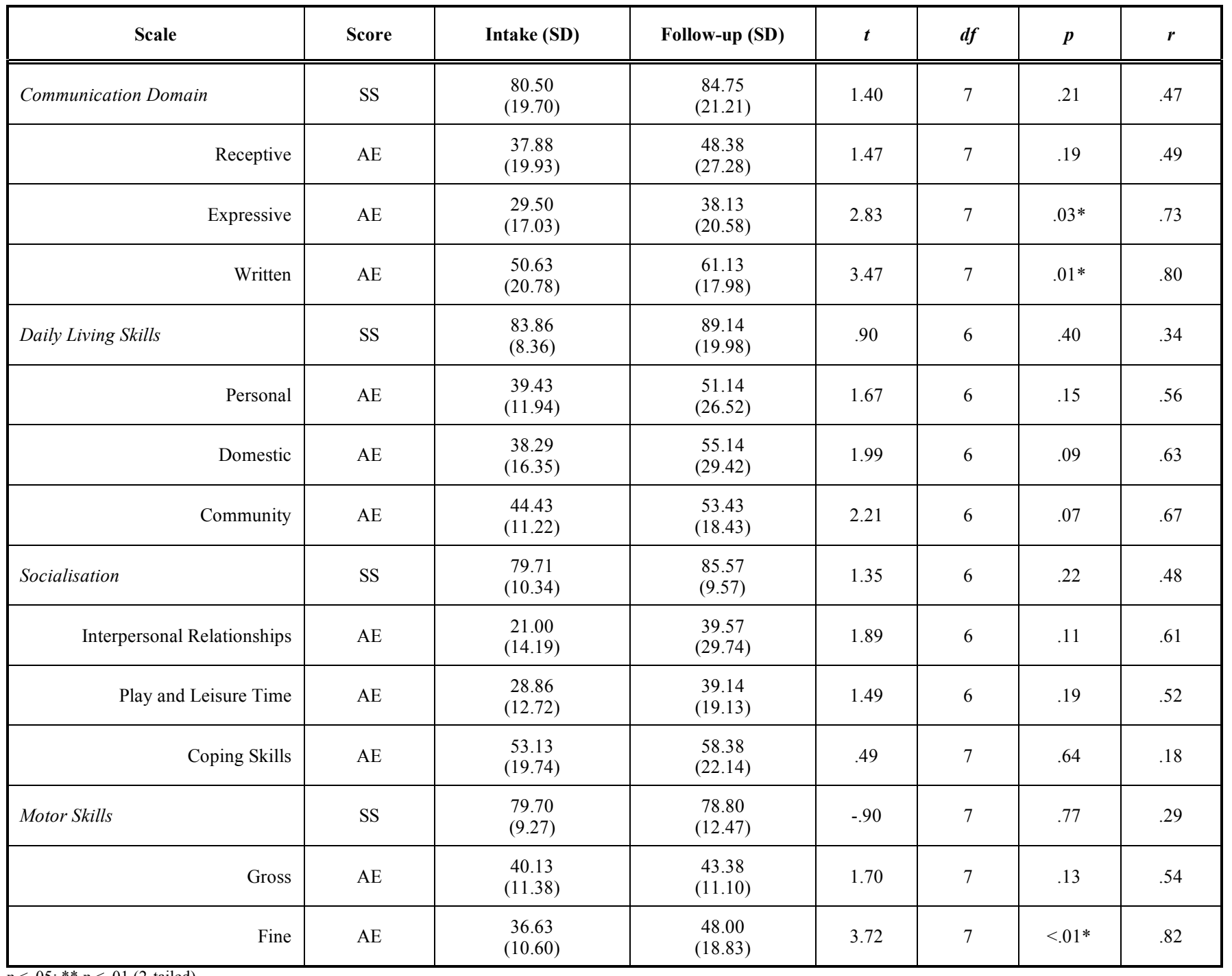


Table 5. VABS-II: Teacher Rating Scale: Age-Equivalents (AE) and Standard Scores (SS)

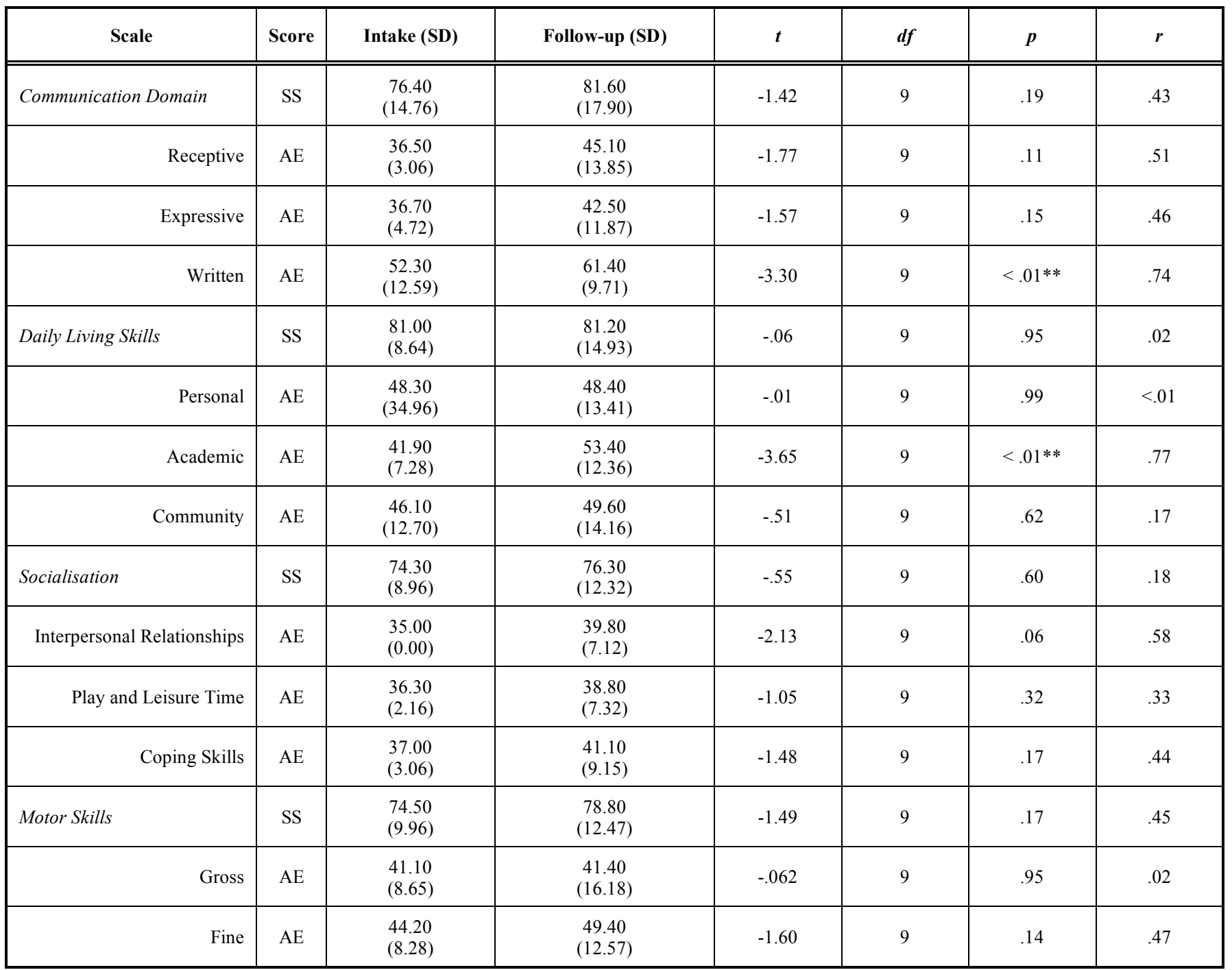

$p<.05 ; * * p<.01$ (2-tailed).

In terms of educational skills, gains were seen in standard scores on the PEP-3 in cognitive/verbal communication, fine and visual-imitation motor skills, motor skills domain score, and social reciprocity. Data indicate that children made gains greater than would be expected by maturation in these areas. These findings are consistent with previous investigations into early learning undertaken by Reed et al., [22, 23] who also found significant gains on the previous edition of the PEP.

In terms of autism symptoms, significant improvements were detected from Time 1 to Time 2 testing on the total score on the SCQ with the average score moving from the clinical range to the non-clinical range. Consistent with this was children's improvements in social reciprocity skills found in the PEP-3. Thus, improvements in ASD symptoms were observed both by parents rated on the SCQ and in the 1:1 assessment. This finding is consistent with those reported by Zachor et al., [26] who found reductions in ASD symptoms on the ADOS social interaction scale for children attending an "eclectic" autism-specific preschool. However, this finding is in contrast to some other evaluations of educationally-based interventions that failed to find improvement in symptoms [22, 23, 27].

In terms of cognitive skills, it was not possible to compare standard scores on the MSEL as some children were beyond the age range of standard scores and, for those in the age range for standard scores, many did not reach the basal level. Age-equivalents were compared with significant gains found in only the receptive language scale. This finding is in line with previous research by Zachor [25] and Ben Itzchak, Lahat, Burgin and Zachor [43] who also found gains only in the receptive language scale when investigating outcomes of an autism-specific preschool which used an "eclectic" model. In common with these researchers, we also argue that the AEIOU teaching curriculum does focus on language themes and practice in related areas (e.g., imitation) which encourage language development.

In terms of adaptive behaviour, significant changes in age-equivalent scores were found in both parents' and teachers' ratings of written communication. In addition, parent ratings indicated significant improvements in expressive communication and fine motor skills. Teacher 
ratings indicated significant improvements in academic skills which were not rated by parents on this measure. Standard scores for domains did not change significantly over the study period. This finding is in line with previous research by Magiati et al., [24] who also found changes only in age-equivalent scores and minimal changes in standard scores on the VABS for children attending an autism-specific eclectic preschool. Lack of developmental progress on standard scores may be due to children in the present study demonstrating more severe impairments at intake than those in similar studies. Consistent with this suggestion, Itzchak and Zachor [44] found that significant progress in adaptive skills was found only for their "mild autism severity group."

A key limitation in this study was the lack of a control or comparison group. However, outcomes in the present study were comparable to those in similar outcome studies in early education and care. In addition, gains on the PEP-3 were made in standard scores compared to a normative sample of children with ASD, which indicates that changes are unlikely to be due to maturation. Thus, although examination of the AEIOU program in comparison to a matched control or comparison group is clearly needed before stronger conclusions can be made, our results remain promising. Future research will compare outcomes of the AEIOU program to other comparable Australian programs that are concurrently collecting data on a similar set of measures at similar intervals.

This pilot study was also limited by a small sample size. Given that medium to large effects were found across many domains, it is likely that power limited our ability to detect significant effects across some areas (e.g., adaptive behaviour). Future research will use larger sample sizes as data are currently being collected on 50 students at Queensland AEIOU programs.

It may be considered a limitation of the present study that, unlike many research programs, that we did not apply exclusion criteria based on IQ, ability, or comorbidity, and children were not randomly assigned to the program, but parents self-selected. In contrast to much of the previous research (for example [45] only included children with IQ scores greater than 50), no child in the present study was excluded based on comorbid diagnosis or low cognitive ability. In addition, our children were referred from a large and diverse socioeconomic group. Any of these factors may have led to a less than favourable outcome. However, given our heterogeneous sample represents a clinical reality, our results may be more likely to generalise to the realworld population of children with ASD who access a similar educationally-based service.

Appropriate measures for assessing change in early intervention posed a challenge in this research. Our key child assessment tools were the MSEL, PEP-3, SCQ and VABS. None of these measures have Australian normative data, although they have been widely used in previous research. In addition, the MSEL norms are for typical development and we found both floor (standard scores) and ceiling (age-equivalents) effects for this population which may have limited our ability to detect significant effects. The PEP-3 does have ASD norms, but these norms are based on a US sample. There is clearly a need for Australian normative data including ASD norms.

Finally, like other educationally-based treatment programs for children with ASD [e.g., 26, 46], we have not identified the active ingredients or critical elements of the program. At this point it is unclear whether instructional intensity (hours), curriculum, parent training, and /or other aspects of the program actually contribute individually or collectively to child outcomes. Delineating the critical elements of the AEIOU treatment program is clearly an important area for follow-up research.

\section{CONCLUSION}

Despite acknowledged limitations, this preliminary evaluation and report does address important questions related to the AEIOU program for children with ASD. First, we have provided information about the various components of this intervention. Second, we have provided new information about the effectiveness of the AEIOU program. Third, preliminary results suggest promising child outcomes, particularly in terms of symptom reduction and increases in educational skills. The present research thus expands the research base into the effectiveness of centre-based educational intervention for children with ASD in Australia and elsewhere.

\section{ACKNOWLEDGEMENTS}

We wish to thank the AEIOU staff who implemented the intervention, to the children who taught us valuable lessons along the way, and the parents who entrusted their children to us and gave their time to complete questionnaires and assessments. Thank you also to the Australian Government Department of Housing, Community Services, and Indigenous Affairs (FaHSCSIA) for their financial support provided for this program.

\section{CONFLICT OF INTEREST DISCLOSURE}

It should be noted that the first author of this report was an employee of the community-based program presented and that benefits could be accrued by that agency if positive findings were generated. This could be seen as motivation for bias or misrepresentation of findings. The authors of this report affirm adherence to the Australian Psychological Society ethical principles in the operation of the agency, provision of treatment services, and presentation of findings.

\section{REFERENCES}

[1] Wray J, Silove N, Knott H. Language disorders and autism. Med J Aust 2005; 182: 354-60.

[2] APA. Diagnostic and statistical manual of mental disorders : DSM-IVTR. 4th, text revision. ed. Washington, DC: American Psychiatric Association 2000

[3] Williams K, MacDermott S, Ridley G, Glasson EJ, Wray JA. The prevalence of autism in Australia. Can it be established from existing data? Aust Paediatr J 2008; 44: 504-10.

[4] MacDermott S, Williams K, Ridley G, Glasson E, Wray J. The prevalence of autism in Australia: Can it be established from existing data?: A report prepared for the Australian Advisory Board on Autism Spectrum Disorders 2006.

[5] Centers for Disease Control and Prevention. Prevalence of Autism Spectrum Disorders. MMWR Surveill Summ 2009; 58:1-20.

[6] Baird G, Simonoff E, Pickles A, et al. Prevalence of disorders of the autism spectrum in a population cohort of children in South Thames: the Special Needs and Autism Project (SNAP). Lancet 2006; 368: 2105.

[7] Kleinman JM, Ventola PE, Pandey J, et al. Diagnostic stability in very young children with autism spectrum disorders. J Autism Child Schizophr 2008; 38: 606-15.

[8] Turner LM, Stone WL, Pozdol SL, Coonrod EE. Follow-up of children with autism spectrum disorders from age 2 to age 9. Autism 2006; 10 : 243-65.

[9] Moore V, Goodson S. How well does early diagnosis of autism stand the test of time? Follow-up study of children assessed for autism at age 
2 and development of an early diagnostic service. Autism 2003; 7: 4763.

[10] Boucher J. The Autistic Spectrum. Characteristics, Causes and Practical Issues. London: Sage 2009.

[11] Mordre M, Groholt B, Knudsen AK, Sponheim E, Mykletun A, Myhre AM. Is Long-Term Prognosis for Pervasive Developmental Disorder Not Otherwise Specified Different from Prognosis for Autistic Disorder? Findings from a 30-Year Follow-Up Study. J Autism Child Schizophr 2011 [Epub ahead of print].

[12] Joshi G, Petty C, Wozniak J, et al. The heavy burden of psychiatric comorbidity in youth with autism spectrum disorders: a large comparative study of a psychiatrically referred population. J Autism Child Schizophr 2010; 40: 1361-70.

[13] Hofvander B, Delorme R, Chaste P, et al. Psychiatric and psychosocial problems in adults with normal-intelligence autism spectrum disorders. BMC Psychiatry 2009; 9: 35

[14] Prior M, Roberts J. Early Intervention for Children with Autism Spectrum Disorders: Guidelines for Best Practice.: Australian Government: Department of Health and Ageing; 2006. Available from: http://www.health.gov.au/internet/main/publishing.nsf/conte nt/D9F44B55D7698467CA257280007A98BD/\$File/autbro.pdf [Accessed: 30/11/11]

[15] Odom S, Boyd B, Hall L, Hume K. Evaluation of Comprehensive Treatment Models for Individuals with Autism Spectrum Disorders. J Autism Child Schizophr 2010; 40: 425-36.

[16] Levy SE, Mandell DS, Schultz RT. Autism. Lancet 2009; 374:1627-38.

[17] Howard JS, Sparkman CR, Cohen HG, Green G, Stanislaw H. A comparison of intensive behavior analytic and eclectic treatments for young children with autism. Res Dev Disabil 2005; 26: 359-83.

[18] Lovaas OI. Behavioral treatment and normal educational and intellectual functioning in young autistic children. J Consult Clin Psychol 1987; 55:3-9.

[19] Frost LA, Bondy AS. The Picture Exchange Communication System Training Manual. Cherry Hill, NJ: PECs, Inc 1994.

[20] Schopler E. A statewide program for the treatment and education of autistic and related communication handicapped children (TEACCH). In: Volkmar F, Ed. Child and Adolescent Clinics of North America: Psychoses and Pervasive Developmental Disorders. Philadelphia: W. B. Sanders 1994; 91-103.

[21] Horner RH. Positive behavior supports. Focus Autism Other Dev Disabl 2000; 15: 97-105.

[22] Reed P, Osborne LA, Corness M. The real-world effectiveness of early teaching interventions for children with autism spectrum disorder. Except Children 2007; 73-417.

[23] Reed P, Osborne LA, Corness M. Effectiveness of special nursery provision for children with autism spectrum disorders. Autism 2010; 14: 67-82.

[24] Magiati I, Charman T, Howlin P. A two-year prospective follow-up study of community-based early intensive behavioural intervention and specialist nursery provision for children with autism spectrum disorders. J Child Psychol Psychiatry 2007; 48: 803-12.

[25] Zachor DA, Ben Itzchak E. Treatment approach, autism severity and intervention outcomes in young children. Res Autism Spectr Disord 2010; 4: 425-32.

[26] Zachor DA, Ben-Itzchak E, Rabinovich A-L, Lahat E. Change in autism core symptoms with intervention. Res Autism Spectr Disord 2007; 1: 304-17.

[27] Charman T, Howlin P, Berry B, Prince E. Measuring Developmental Progress of Children with Autism Spectrum Disorder on School Entry Using Parent Report. Autism 2004; 8: 89-100.

[28] Roberts J, Prior M. A review of the research to identify the most effective models of practice in early intervention for children with autism spectrum disorders. Australian Government Department of Health and Ageing, Australia [serial on the Internet]. 2006: Available from: www.health.gov.au/internet/main/publishing.nsf/ Content/846 804F6D67F34F3CA257280007853DE/\$File/autrev.pdf [Accessed: $30 / 11 / 11]$

[29] National Autism Center. National Standards Report 2009: Available from: http://www.nationalautismcenter.org/pdf/NAC $\%$ 20Standards\% 20Report.pdf

[30] American Psychiatric Association. American Psychiatric Association DSM-5 Development: Proposed Revision: 299.00 Autistic Disorder. Arlington, VA: American Psychiatric Association; 2010. Available from: http://www.dsm5.org/ProposedRevisions/Pages/proposedrevision .aspx?rid=94\# [Accessed: 30/11/11].

[31] Rutter M, Bailey A, Lord C. Social Communication Questionnaire. Los Angeles, CA: Western Psychological Services 2003.

[32] Lee LC, David AB, Rusyniak J, Landa R, Newschaffer CJ. Performance of the Social Communication Questionnaire in children receiving preschool special education services. Res Autism Spectr Disord 2007; 1: 126-38.

[33] Schopler E, Lansing MD, Reichler RJ, Marcus LM. Examiner's manual of the Psychoeducational Profile (3rd ed.). Austin, TX: Pro-ed Incorporation 2005

[34] Mullen EM. Mullen Scales of Early Learning (AGS ed.). Circle Pines, MN: American Guidance Service Inc 1995.

[35] Akshoomoff N. Use of the Mullen Scales of Early Learning for the Assessment of Young Children with Autism Spectrum Disorders. Child Neuropsychol 2006; 12:269 - 77.

[36] Itzchak EB, Zachor DA. Who benefits from early intervention in autism spectrum disorders? Res Autism Spectr Disord 2011; 5: 345-50.

[37] Keen D, Couzens D, Muspratt S, Rodger S. The effects of a parentfocused intervention for children with a recent diagnosis of autism spectrum disorder on parenting stress and competence. Res Autism Spectr Disord 2010; 4: 229-41.

[38] Berument S, Rutter M, Lord C, Pickles A, Bailey A. Autism screening questionnaire: diagnostic validity. Br J Psychiat 1999; 175: 444-51.

[39] Sparrow S, Dominic V, Cicchetti DA, Balla DA. Vineland Adaptive Behavior Scales (2nd ed.). Circle Pines, MN: American Guidance Service 2005.

[40] Lord C, Rutter M, Le Couteur A. Autism Diagnostic InterviewRevised: A revised version of a diagnostic interview for caregivers of individuals with possible pervasive developmental disorders. J Autism Child Schizophr 1994; 24: 659-85.

[41] Carter, AS, Volkmar, FR, Sparrow, SS, et al.The Vineland Adaptive Behavior Scales: Supplementary Norms for Individuals with Autism. J Autism Child Schizophr 1998; 28(4): 287-302.

[42] Klin A, Carter AS, Sparrow SS. Psychological assessment of children with autism. In: Cohen DJ, Volkmar FR, editors. Handbook of Autism and Pervasive Developmental Disorders. 2nd ed. New York: Wiley 1997. p. 418-27.

[43] Itzchak EB, Lahat E, Burgin R, Zachor AD. Cognitive, behavior and intervention outcome in young children with autism. Res Dev Disabil 2008; 29:447-58.

[44] Itzchak EB, Zachor DA. Who benefits from early intervention in autism spectrum disorders? Res. Autism Spectr. Disord 2011; 5: 345-50.

[45] Eikeseth S, Smith T, Jahr E, Eldevik S. Intensive Behavioral Treatment at School for 4- to 7-Year-Old Children with Autism. Behav Modif 2002; 26: 49-68.

[46] Charman T, Howlin P, Aldred C, et al. Research into early intervention for children with autism and related disorders: methodological and design issues. Report on a workshop funded by the Wellcome Trust, Institute of Child Health, London, UK, November 2001. Autism 2003; 7:217-25. 